\title{
Información básica sobre recursos y servicios del Sistema Nacional de Salud, 1994-2000
}

$\mathrm{C}$ omo en años anteriores, la Dirección General de Estadística e Informática de la Secretaría de Salud, encargada de la integración y difusión de estadísticas sectoriales, presenta en nueve tabulados la información más relevante sobre recursos y servicios que han generado las diversas instituciones del Sistema Nacional de Salud durante la presente administración.

Estos tabulados tienen como propósito ofrecer un panorama general de la información básica disponible en las diversas instituciones que conforman el Sistema Nacional de Salud, durante el periodo 19942000, con el fin de que constituyan un medio para conocer las condiciones de salud en México en la administración que concluye.

La información de este documento fue recopilada e integrada con datos generados por las siguientes instituciones: Secretaría de Salud, Instituto Mexicano del Seguro Social, Instituto de Seguridad y Ser- vicios Sociales de los Trabajadores del Estado, Servicios Médicos del Departamento del Distrito Federal, Petróleos Mexicanos, Secretaría de la Defensa Nacional y Secretaría de Marina. Esta tarea ha sido realizada en el seno del Grupo Interinstitucional de Información, instancia conformada en 1983 y que reúne a los representantes de las diversas instituciones ya mencionadas, con el propósito de generar y difundir información sectorial homogénea, confiable y oportuna.

La información se ha estructurado en ocho cuadros en donde se presenta la información de las principales variables sobre recursos físicos, materiales y humanos con que cuentan las instituciones, así como los principales servicios otorgados, por último se presenta un cuadro sobre las principales causas de mortalidad.

Resulta indispensable hacer la aclaración respecto de las variables incluidas en los cuadros de recursos y servicios. Estas han sido difundidas a través de la publicación anual del Sistema Nacional de Salud: Boletín de Información Estadística (números 14 al 19); sin embargo, las cifras ahora publicadas difieren en muchos casos debido a una revisión realizada recientemente, con fines de validación y actualización, por las diversas instituciones involucradas.

Para mayor información sobre recursos y servicios puede consultar el Boletín de Información Estadística en particular los números 14 al 19 del volumen I, así como los Anuarios Estadísticos del mismo periodo, ambos editados por la Dirección General de Estadística e Informática. La consulta puede hacerse en el Centro de Referencia de Información Estadística en Salud (CREIS), ubicado en Homero No. 213, 1er. piso, colonia Chapultepec Morales, 11570 México, D.F. 
Cuadro I

Infraestructura médica del Sistema Nacional de Salud. Unidades médicas según tipo. MÉXICO, 1994-2000

\begin{tabular}{|c|c|c|c|c|c|c|c|}
\hline Concepto & 1994 & 1995 & 1996 & 1997 & 1998 & 1999 & $2000 *$ \\
\hline Total de unidades médicas & 14672 & 15172 & 15653 & 16477 & 17135 & 17822 & 18557 \\
\hline Población abierta & 11144 & 11645 & 12063 & 12814 & 13439 & 14102 & 14796 \\
\hline SSA y SS de entidad federativa ${ }^{\ddagger}$ & 7508 & 8002 & 8312 & 9207 & 9832 & 10493 & 11187 \\
\hline DDF & 143 & 137 & 144 & - & - & - & - \\
\hline IMSS-Solidaridad & 3493 & 3506 & 3607 & 3607 & 3607 & 3609 & 3609 \\
\hline Población derechohabiente & 3528 & 3527 & 3590 & 3663 & 3696 & 3720 & 376 \\
\hline IMSS & 747 & 748 & 752 & 755 & 1775 & 784 & 1819 \\
\hline ISSSTE & 1192 & 1190 & 1198 & 1212 & 1220 & 1232 & 1236 \\
\hline Pemex & 153 & $|5|$ & 202 & 206 & 219 & 219 & 215 \\
\hline SDN & 277 & 279 & 279 & 330 & 349 & 357 & 363 \\
\hline SM & 159 & 159 & 159 & 160 & 133 & 128 & 128 \\
\hline De consulta externa & 13897 & 14378 & 14859 & 15632 & 16246 & 16900 & 17590 \\
\hline Población abierta & 10802 & 1129 & 11 701 & 12405 & 12995 & 13628 & 14296 \\
\hline SSA y SS de entidad federativa ${ }^{\ddagger}$ & 7256 & 7744 & 8049 & 8866 & 9456 & 10088 & 10756 \\
\hline DDF & 112 & 106 & 113 & - & - & - & - \\
\hline IMSS-Solidaridad & 3434 & 3441 & 3539 & 3539 & 3539 & 3540 & 3540 \\
\hline Población derechohabiente & 3095 & 3087 & 3158 & 3227 & 3251 & 3272 & 3294 \\
\hline IMSS & 1482 & 482 & 496 & 1499 & 1518 & 1527 & 1552 \\
\hline TSSSTE & 1104 & 1098 & 104 & 1114 & 1121 & 1132 & 1133 \\
\hline Pemex & $|3|$ & 128 & 179 & 183 & 196 & 196 & 192 \\
\hline SDN & 247 & 248 & 248 & 299 & 313 & 321 & 321 \\
\hline SM & $|3|$ & $|3|$ & $|3|$ & 132 & 103 & 96 & 96 \\
\hline De hospitalización ${ }^{\S}$ & 775 & 794 & 794 & 845 & 889 & 922 & 967 \\
\hline Población abierta & 342 & 354 & 362 & 409 & 444 & 474 & 500 \\
\hline SSA y SS de entidad federativa ${ }^{\ddagger}$ & 252 & 258 & 263 & 341 & 376 & 405 & 431 \\
\hline DDF & 31 & 31 & 31 & - & - & - & - \\
\hline TMSS-Solidaridad & 59 & 65 & 68 & 68 & 68 & 69 & 69 \\
\hline Población derechohabiente & 433 & 440 & 432 & 436 & 445 & 448 & 467 \\
\hline IMSS & 265 & 266 & 256 & 256 & 257 & 257 & 267 \\
\hline TSSSTE & 88 & 92 & 94 & 98 & 99 & 100 & 103 \\
\hline Pemex & 22 & 23 & 23 & 23 & 23 & 23 & 23 \\
\hline SDN & 30 & 31 & 31 & 31 & 36 & 36 & 42 \\
\hline SM & 28 & 28 & 28 & 28 & 30 & 32 & 32 \\
\hline General & 640 & 661 & 654 & 696 & 734 & 765 & 807 \\
\hline Población abierta & 270 & 284 & 285 & 323 & 352 & 379 & 404 \\
\hline SSA y SS de entidad federativa ${ }^{\ddagger}$ & 198 & 206 & 204 & 255 & 284 & 310 & 335 \\
\hline DDF & 13 & 13 & 13 & - & - & - & - \\
\hline IMSS-Solidaridad & 59 & 65 & 68 & 68 & 68 & 69 & 69 \\
\hline Población derechohabiente & 370 & 377 & 369 & 373 & 382 & 386 & 403 \\
\hline IMSS & 224 & 225 & 215 & 215 & 216 & 217 & 226 \\
\hline ISSSTE & 77 & 81 & 83 & 87 & 88 & 89 & 92 \\
\hline Pemex & 14 & 15 & 15 & 15 & 15 & 15 & 15 \\
\hline SDN & 28 & 29 & 29 & 29 & 34 & 34 & 39 \\
\hline SM & 27 & 27 & 27 & 27 & 29 & 31 & 31 \\
\hline De especialidad & 135 & 133 & 140 & 149 & 155 & 157 & 160 \\
\hline Población abierta & 72 & 70 & 77 & 86 & 92 & 95 & 96 \\
\hline SSA y SS de entidad federativa ${ }^{\ddagger}$ & 54 & 52 & 59 & 86 & 92 & 95 & 96 \\
\hline DDF & 18 & 18 & 18 & - & - & - & - \\
\hline TMSS-Solidaridad & 0 & 0 & 0 & 0 & 0 & 0 & 0 \\
\hline Población derechohabiente & 63 & 63 & 63 & 63 & 63 & 62 & 64 \\
\hline TMSS & 41 & 41 & 41 & 41 & 41 & 40 & 41 \\
\hline ISSSTE & $\Pi$ & $\Pi$ & $\pi$ & $\pi$ & $\pi$ & $\Pi$ & $\pi$ \\
\hline Pemex & 8 & 8 & 8 & 8 & 8 & 8 & 8 \\
\hline SDN & 2 & 2 & 2 & 2 & 2 & 2 & 3 \\
\hline $\mathrm{SM}$ & 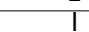 & $T$ & 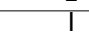 & 2 & 2 & 2 & \\
\hline
\end{tabular}

* Cifras estimadas

¥ Hasta 1996 sólo incluye información de la SSA

$\S$ Incluye generales y de especialidad

SSA: Secretaría de Salud; DDF: Departamento del Distrito Federal; IMSS-Solidaridad: Instituto Mexicano del Seguro Social Régimen de Solidaridad; IMSS: Instituto Mexicano del Seguro Social Régimen de Seguridad Social; ISSSTE: Instituto de Seguridad y Servicios Sociales de los Trabajadores del Estado; Pemex: Petróleos Mexicanos; SDN: Secretaría de la Defensa Nacional; SM: Secretaría de Marina

Fuente: Secretaría de Salud, a través de la información proporcionada por las instituciones del Sistema Nacional de Salud 


\section{Cuadro II}

\section{Principales recursos materiales. Sistema Nacional de Salud, México, 1994-2000}

\begin{tabular}{|c|c|c|c|c|c|c|c|}
\hline Concepto & 1994 & 1995 & 1996 & 1997 & 1998 & 1999 & $2000 *$ \\
\hline Camas censables & 66692 & 67565 & 67997 & 70367 & 72912 & 73701 & 75611 \\
\hline Población abierta & 27029 & 27510 & 27650 & 30331 & 32708 & 33224 & 33738 \\
\hline SSA y SS de entidad federativa ${ }^{\ddagger}$ & 23160 & 23672 & 23674 & 28483 & 30770 & 31252 & 31766 \\
\hline DDF & 2111 & 2115 & 2145 & - & - & - & - \\
\hline IMSS-Solidaridad & 1758 & 1723 & $|83|$ & 1848 & 1938 & 1972 & 1972 \\
\hline Población derechohabiente & 39663 & 40055 & 40347 & 40036 & 40204 & 40477 & 41873 \\
\hline IMSS & 28012 & 28294 & 28230 & 28226 & 28118 & 28334 & 29374 \\
\hline ISSSTE & 6465 & 6596 & 6744 & 6824 & 6823 & 6746 & 6777 \\
\hline Pemex & 1101 & 1074 & 1072 & 1072 & $|06|$ & 954 & 954 \\
\hline SDN & 3483 & 3469 & 3625 & 3200 & 3488 & 3703 & 4028 \\
\hline SM & 602 & 622 & 676 & $7 \mid 4$ & $7 \mid 4$ & 740 & 740 \\
\hline Consultorios & 40528 & 41769 & 42747 & 45536 & 46836 & 47973 & 49433 \\
\hline Población abierta & 20407 & 21437 & 22503 & 24299 & 25295 & 26395 & 27392 \\
\hline SSA y SS de entidad federativa ${ }^{\ddagger}$ & 15962 & 16917 & 17872 & 20111 & 21112 & 22202 & 23199 \\
\hline DDF & 451 & 466 & 449 & - & - & - & - \\
\hline TMSS-Solidaridad & 3994 & 4054 & 4182 & 4188 & 4183 & 4193 & 4193 \\
\hline Población derechohabiente & 20121 & 20332 & 20244 & 21237 & $2154 \mid$ & 21578 & 22041 \\
\hline IMSS & 13085 & 13204 & 13233 & 13259 & 13437 & 13660 & 13988 \\
\hline ISSSTE & 4805 & 4888 & 5000 & 5047 & 5086 & 5164 & 5221 \\
\hline Pemex & 1398 & 1398 & 1169 & 447 & 1447 & 1252 & 1252 \\
\hline SDN & 526 & 535 & 535 & 1103 & 1185 & 1112 & 1190 \\
\hline SM & 307 & 307 & 307 & 381 & 386 & 390 & 390 \\
\hline Quirófanos & 2220 & 2250 & 2266 & 2385 & 2511 & 2568 & 2657 \\
\hline Población abierta & 884 & 904 & 913 & 995 & 1107 & 1154 & 1181 \\
\hline SSA y SS de entidad federativa ${ }^{\ddagger}$ & 757 & 768 & 776 & 927 & 1039 & 1085 & 1112 \\
\hline DDF & 68 & 71 & 69 & - & - & - & - \\
\hline IMSS-Solidaridad & 59 & 65 & 68 & 68 & 68 & 69 & 69 \\
\hline Población derechohabiente & 1336 & 1346 & 1353 & 1390 & 1404 & 1414 & 476 \\
\hline IMSS & 921 & 943 & 939 & 937 & 935 & 951 & 999 \\
\hline ISSSTE & 255 & 266 & 278 & 283 & 285 & 284 & 289 \\
\hline Pemex & 68 & 68 & 66 & 60 & 63 & 58 & 55 \\
\hline SDN & 62 & 38 & 38 & 71 & 78 & 77 & 89 \\
\hline SM & 30 & 31 & 32 & 39 & 43 & 44 & 44 \\
\hline Laboratorio de análisis clínicos & | 44 | & 1499 & 1513 & 1602 & 1690 & | 727 & 1779 \\
\hline Población abierta & 787 & 806 & 830 & 885 & 945 & 962 & 972 \\
\hline SSA y SS de entidad federativa ${ }^{\ddagger}$ & 697 & 710 & 732 & 817 & 877 & 893 & 903 \\
\hline DDF & 31 & 31 & 30 & - & - & - & - \\
\hline IMSS-Solidaridad & 59 & 65 & 68 & 68 & 68 & 69 & 69 \\
\hline Población derechohabiente & 654 & 693 & 683 & 717 & 745 & 765 & 807 \\
\hline IMSS & 445 & 456 & 466 & 468 & 476 & 481 & 511 \\
\hline ISSSTE & 132 & $|6|$ & 139 & 170 & 184 & 196 & 202 \\
\hline Pemex & 22 & 22 & 22 & 22 & 22 & 22 & 22 \\
\hline SDN & 30 & 29 & 29 & 30 & 35 & 37 & 43 \\
\hline SM & 25 & 25 & 27 & 27 & 28 & 29 & 29 \\
\hline Gabinete de rayos $X$ & 2176 & 2235 & 2309 & 2398 & 2467 & 2558 & 2638 \\
\hline Población abierta & 908 & 933 & 982 & 1041 & 1140 & 1176 & 1187 \\
\hline SSA y SS de entidad federativa ${ }^{\ddagger}$ & 778 & 794 & 843 & 973 & 1072 & I 107 & 1118 \\
\hline DDF & 71 & 74 & 71 & - & - & - & - \\
\hline IMSS-Solidaridad & 59 & 65 & 68 & 68 & 68 & 69 & 69 \\
\hline Población derechohabiente & 1268 & 1302 & 1327 & 1357 & 1 327 & 1382 & 451 \\
\hline IMSS & 792 & 813 & 825 & 825 & 836 & 845 & 902 \\
\hline ISSSTE & 369 & 405 & 404 & 419 & 404 & 440 & 446 \\
\hline Pemex & 26 & 26 & 39 & 51 & 23 & 32 & 32 \\
\hline SDN & 54 & 30 & 30 & 33 & 38 & 38 & 44 \\
\hline SM & 27 & 28 & 29 & 29 & 26 & 27 & 27 \\
\hline
\end{tabular}

\footnotetext{
* Cifras estimadas
}

¥ Hasta 1996 sólo incluye información de la SSA

SSA: Secretaría de Salud; DDF: Departamento del Distrito Federal; IMSS-Solidaridad: Instituto Mexicano del Seguro Social Régimen de Solidaridad; IMSS: Instituto Mexicano del Seguro Social Régimen de Seguridad Social; ISSSTE: Instituto de Seguridad y Servicios Sociales de los Trabajadores del Estado; Pemex: Petróleos Mexicanos; SDN: Secretaría de la Defensa Nacional; SM: Secretaría de Marina 


\section{Médicos y enfermeras. Sistema Nacional de Salud, México, I 994-2000}

\begin{tabular}{|c|c|c|c|c|c|c|c|}
\hline Concepto & 1994 & 1995 & 1996 & 1997 & 1998 & 1999 & $2000 *$ \\
\hline Total de médicos y enfermeras & 259074 & 267097 & 274100 & 285432 & 299447 & 307031 & 310103 \\
\hline Población abierta & 96957 & 101637 & 106732 & 116112 & 126816 & 132375 & 134659 \\
\hline SSA y SS de entidad federativa ${ }^{\ddagger}$ & 79310 & 83548 & 88676 & 104000 & 114557 & 119987 & $12227 \mid$ \\
\hline DDF & 6049 & 5978 & 5894 & - & - & - & - \\
\hline IMSS-Solidaridad & II 598 & 12111 & 12162 & 12112 & 12259 & 12388 & 12388 \\
\hline Población derechohabiente & 162117 & 165460 & 167368 & 169320 & 172631 & 174656 & 175444 \\
\hline IMSS & 118368 & 120207 & 121442 & 123339 & 126168 & 127266 & 127266 \\
\hline ISSSTE & 32802 & 33838 & 34148 & 34947 & 35892 & 36544 & 37210 \\
\hline Pemex & 5204 & 5100 & 5041 & 5059 & 5061 & 5007 & 5007 \\
\hline SDN & 4259 & 4815 & 5237 & 4230 & 3947 & 4051 & 4173 \\
\hline SM & 1484 & 1500 & 1500 & 1745 & 1563 & 1788 & 1788 \\
\hline Médicos $§$ & 106896 & 110839 & II4 I44 & 121306 & 126685 & 128957 & 130469 \\
\hline Población abierta & 40826 & 43165 & 45664 & 51095 & 54953 & 55850 & 56971 \\
\hline SSA y SS de entidad federativa $a^{\ddagger}$ & 32889 & 35033 & 37620 & 45676 & 49453 & 50309 & 51430 \\
\hline DDF & 2758 & 2747 & 2610 & - & - & - & - \\
\hline IMSS-Solidaridad & 5179 & 5385 & 5434 & 5419 & 5500 & 5541 & 5541 \\
\hline Población derechohabiente & 66070 & 67674 & 68480 & 70211 & 71732 & 73107 & 73498 \\
\hline IMSS\# & $46|5|$ & 47420 & 47813 & 48658 & 50017 & 50764 & 50764 \\
\hline ISSSTE & 15311 & 15775 & 15945 & 16487 & 16948 & 17309 & 17680 \\
\hline Pemex & 2510 & 2364 & 2392 & 2405 & 2403 & 2355 & 2355 \\
\hline SDN & 445 & 1446 & $166 \mid$ & 1829 & 1728 & 1873 & 1893 \\
\hline SM & 653 & 669 & 669 & 832 & 636 & 806 & 806 \\
\hline Enfermeras $^{\&}$ & $|52| 78$ & 156258 & 159956 & 164126 & 172762 & 178074 & 179634 \\
\hline Población abierta & $56|3|$ & 58472 & 61068 & 65017 & 71863 & 76525 & 77688 \\
\hline SSA y SS de entidad federativa $a^{\ddagger}$ & 46421 & 48515 & 51056 & 58324 & 65104 & 69678 & 70841 \\
\hline DDF & 3291 & 3231 & 3284 & - & - & - & - \\
\hline IMSS-Solidaridad & 6419 & 6726 & 6728 & 6693 & 6759 & 6847 & 6847 \\
\hline Población derechohabiente & 96047 & 97786 & 98888 & 99109 & 100899 & 101549 & 101946 \\
\hline IMSS & 72217 & 72787 & 73629 & 74681 & $76|5|$ & 76502 & 76502 \\
\hline ISSSTE & $1749 \mid$ & 18063 & 18203 & 18460 & 18944 & 19235 & 19530 \\
\hline Pemex & 2694 & 2736 & 2649 & 2654 & 2658 & 2652 & 2652 \\
\hline SDN & 2814 & 3369 & 3576 & 2401 & 2219 & 2178 & 2280 \\
\hline SM & 831 & 831 & 831 & 913 & 927 & 982 & 982 \\
\hline
\end{tabular}

* Cifras estimadas

‡ Hasta 1996 sólo incluye información de la SSA

$\S$ Comprende a los médicos generales, especialistas, pasantes, odontólogos y personal médico en otras labores

\# Incluye médicos que laboran en oficinas centrales: 1995, 124, y 1996, 127

\& Comprende a las enfermeras auxiliares, generales, especializadas y otras

SSA: Secretaría de Salud; DDF: Departamento del Distrito Federal; IMSS-Solidaridad: Instituto Mexicano del Seguro Social Régimen de Solidaridad; IMSS: Instituto Mexicano del Seguro Social Régimen de Seguridad Social; ISSSTE: Instituto de Seguridad y Servicios Sociales de los Trabajadores del Estado; Pemex: Petróleos Mexicanos; SDN: Secretaría de la Defensa Nacional; SM: Secretaría de Marina

Fuente: Secretaría de Salud, a través de la información proporcionada por las instituciones del Sistema Nacional de Salud 


\section{Consulta externa otorgada. Sistema Nacional de Salud, México, 1994-2000}

Concepto

1994

1995

1996

1997

1998

1999

$2000 *$

Total de consultas

$167256981 \quad 180969695$

$89986833 \quad 202029656$

$11058133 \quad 225791285$

238878821 Población abierta $50483294 \quad 55840038$ $58085923 \quad 6640566$ 74091405 85770723 92008501

\begin{tabular}{lr}
\hline SSA y SS de entidad federativa $^{\ddagger}$ & 39774641 \\
\hline DDF & 2254013 \\
\hline IMSS-Solidaridad & 8454640 \\
\hline Población derechohabiente & 11673687 \\
\hline IMSS & 90746287 \\
\hline ISSSTE & 19048444 \\
\hline Pemex & 4348265 \\
\hline SDN & 1992834 \\
\hline SM & 637857
\end{tabular}

43811872

44854109

54119303
60190774 2329217 235094

9698949

2350941

125129657

131900910

12286358

13900631 70582164 75222600

96830889

99744814

135623995

136966728

15188559

16785901

20791682 22090219

103269673

$103901186 \quad 106789885$

4773048

5482020

23268579

24000342

106789885

146870320

2038326

3701594

5596518

5185200

24219645

112162534

637857

695712

882263

917797

2727755

054048

24836670

$113023812 \quad 122495991$

28177007

38057072

1152245

I 019623

5650000

Generales $37637411 \quad 41871754$ 43463707 4978662

146351372

157857024 3100758 Población abierta $29703789 \quad 32856655$ SSA y SS de entidad federativa ${ }^{\ddagger}$ 482832 DDF 7450790 493560 IMSS-Solidaridad 75386401 8521539

33396403

38852774 483737

Población derechohabiente IMSS

59536605

80624237

9583567

10933850

66905182

$16775379 \mid$

ISSSTE

12764773

63512217

84713300

88270448

$5316 \mid 778$

71 926460

Pemex

I 925857

3918075

65601841

68784211

12453925

13743404 56699900 SDN 914035

2161050 14784942 15553399

15226560

$245|3|$

232201

490884

I 54| 096

2563354

70812684

95827331

$16051192 \quad 16128309$

74866727

6534742

294537

I 089882

2306094

2300204

2599000

279602

414150

I 299049

I 374225

De especialidad

26091419

27929431

29636697

31 543346

3184957 |

33308809

4

Población abierta
SSA y SS de

5536183

SSA y SS de entidad federativa ${ }^{\ddagger}$

569132

5901842

6129723

7820088

7712099

8556485

34085 I7I DDF 260958

548005

5277807

747076

MSS-Solidaridad

20555236

312570

517409

334507

7309088

$8157 \mid 17$

9049322

Población derechohabiente

13740284

22027589

23506974

349327

403011

399368

$-$

IMSS

ISSSTE

4428779

4389143

14645013

23723258

24137472

24752324

426222

Pemex

| 54 | 754

4880754

5200918

14883012

15169217

15804249

25035849

SDN 578433

| 71575 |

I 937817

5509376

5678551

$584 \mid 277$

15579267

265986

706601

I 308574

I 899745

| $84 \mid 753$

$607 \mid 423$

335340

414652

438698

484290

911772

034000

30544273

32173129

32429238

353273

387492

\begin{tabular}{|c|c|c|c|c|c|c|c|}
\hline Otras $\S$ & $28|4| 750$ & 30544273 & $32 \quad 173 \quad 129$ & 32429238 & 32857190 & 34625452 & 37039859 \\
\hline Población abierta & 7309700 & 8066442 & 8492493 & 8798949 & 9368751 & 10309056 & 11032719 \\
\hline SSA y SS de entidad federativa ${ }^{\ddagger}$ & 5364759 & 5913950 & 6179899 & 7795768 & 8325056 & 9263269 & 9899600 \\
\hline DDF & I 202049 & I 287652 & I 349795 & - & - & - & - \\
\hline IMSS-Solidaridad & 742892 & 864840 & 962799 & $|003| 8 \mid$ & 1043695 & I 045787 & 1133119 \\
\hline Población derechohabiente & 20832050 & 22477831 & 23680636 & 23630289 & 23488439 & 24316396 & $26007 \quad 140$ \\
\hline IMSS & 17469398 & 18929529 & 19497960 & 19602450 & 19381508 & 20172952 & 21716540 \\
\hline ISSSTE & 1854892 & 1992853 & 2104359 & 2205804 & 2270599 & 2250059 & 2230505 \\
\hline Pemex & 880654 & 896247 & 1053319 & I 049704 & 979361 & 912091 & 1017000 \\
\hline SDN & 500366 & $53 \mid 031$ & 851924 & 572834 & 603166 & 726540 & 762866 \\
\hline SM & 126740 & $|28| 7 \mid$ & 173074 & 199497 & 253805 & 254754 & 280229 \\
\hline
\end{tabular}

* Cifras estimadas

\# Hasta 1996 sólo incluye información de la SSA

$\S$ Incluye las consultas de urgencias y odontológicas

SSA: Secretaría de Salud; DDF: Departamento del Distrito Federal; IMSS-Solidaridad: Instituto Mexicano del Seguro Social Régimen de Solidaridad; IMSS: Instituto Mexicano del Seguro Social Régimen de Seguridad Social; ISSSTE: Instituto de Seguridad y Servicios Sociales de los Trabajadores del Estado; Pemex: Petróleos Mexicanos; SDN: Secretaría de la Defensa Nacional; SM: Secretaría de Marina

Fuente: Secretaría de Salud, a través de la información proporcionada por las instituciones del Sistema Nacional de Salud 


\section{Principales servicios de hospitalización. Sistema Nacional de Salud, México, 1994-2000}

\begin{tabular}{|c|c|c|c|c|c|c|c|}
\hline Concepto & 1994 & 1995 & 1996 & 1997 & 1998 & 1999 & $2000^{*}$ \\
\hline Intervenciones quirúrgicas & 2174276 & 2316064 & 2400801 & 2482288 & 2576405 & 2668618 & 2750282 \\
\hline Población abierta & 646771 & 710558 & 748775 & 814554 & 874660 & 951181 & 999923 \\
\hline SSA y SS de entidad federativa ${ }^{\ddagger}$ & 515456 & 564601 & 586440 & 699081 & 753784 & 833857 & 885200 \\
\hline DDF & 45284 & 46062 & 43771 & - & - & - & - \\
\hline IMSS-Solidaridad & 86031 & 99895 & 118564 & 115473 & 120876 & 117324 & 114723 \\
\hline Población derechohabiente & I 527505 & I 605506 & 1652026 & I 667734 & I $70 \mid 745$ & 1717437 & 1750359 \\
\hline IMSS & I 239808 & 1293319 & I 326724 & I 349830 & I 366847 & 1389566 & 1417357 \\
\hline ISSSTE & 217916 & 236649 & 256950 & 253270 & 262681 & 258076 & 261947 \\
\hline Pemex & 36704 & 30877 & 32368 & 30768 & 26990 & 30498 & 27000 \\
\hline SDN & 23250 & $35|4|$ & 27063 & 24005 & 31112 & 29959 & 33792 \\
\hline SM & 9827 & 9520 & 8921 & 9861 & 14115 & 9338 & 10263 \\
\hline Egresos & 3720933 & 3813933 & 3842076 & 3996515 & 4003103 & 4156862 & 4281463 \\
\hline Población abierta & | 307 | 22 & I 409877 & I 443057 & I 577690 & | 64803 | & 1763636 & $|863| 40$ \\
\hline SSA y SS de entidad federativa ${ }^{\ddagger}$ & 1028455 & I 108064 & 1126095 & I 354282 & $|4| 6 \mid 92$ & 1527689 & 1620500 \\
\hline DDF & 105643 & 104250 & 97428 & - & - & - & - \\
\hline IMSS-Solidaridad & 173024 & 197563 & 219534 & 223408 & 231839 & 235947 & 242640 \\
\hline Población derechohabiente & 2413811 & 2404056 & 2399019 & 2418825 & 2355072 & 2393226 & 2418323 \\
\hline IMSS & I 930347 & I 907275 & I 879356 & | 89| 201 & I 834794 & 1863126 & 1878519 \\
\hline ISSSTE & 322948 & 339701 & 353035 & 360369 & 358427 & 350814 & 356076 \\
\hline Pemex & 81535 & 70078 & 74405 & 73246 & 62641 & 66118 & 62500 \\
\hline SDN & 59747 & 67703 & 70496 & 69291 & 74945 & 88816 & 94452 \\
\hline SM & 19234 & 19299 & 21727 & 24718 & 24265 & 24352 & 26776 \\
\hline
\end{tabular}

* Cifras estimadas

‡ Hasta 1996 sólo incluye información de la SSA

SSA: Secretaría de Salud; DDF: Departamento del Distrito Federal; IMSS-Solidaridad: Instituto Mexicano del Seguro Social Régimen de Solidaridad; IMSS: Instituto Mexicano del Seguro Social Régimen de Seguridad Social; ISSSTE: Instituto de Seguridad y Servicios Sociales de los Trabajadores del Estado; Pemex: Petróleos Mexicanos; SDN: Secretaría de la Defensa Nacional; SM: Secretaría de Marina

Fuente: Secretaría de Salud, a través de la información proporcionada por las instituciones del Sistema Nacional de Salud 


\section{Cuadro VI}

\section{Acciones de planificación familiar. Sistema Nacional de Salud, México, 1994-2000}

\begin{tabular}{|c|c|c|c|c|c|c|c|}
\hline Concepto & 1994 & 1995 & 1996 & 1997 & 1998 & 1999 & $2000^{*}$ \\
\hline Número de consultas de planificación familiar & 7869638 & 9271105 & 9690655 & 9242824 & 9332742 & 9656646 & 9975687 \\
\hline Población abierta & 3776170 & 5207918 & 5827567 & 5185428 & 5366912 & 5700729 & 6066900 \\
\hline SSA y SS de entidad federativa ${ }^{\ddagger}$ & 3720284 & 4212157 & 4780412 & 5185428 & 5366912 & 5700729 & 6066900 \\
\hline DDF & 55886 & 59485 & 58619 & - & - & - & - \\
\hline IMSS-Solidaridad & ND & 936276 & 988536 & ND & ND & ND & ND \\
\hline Población derechohabiente & 4093468 & $4063 \quad 187$ & 3863088 & 4057396 & 3965830 & 3955917 & 3908787 \\
\hline IMSS & 3226962 & 3202058 & 2929508 & 3146775 & 3084074 & 2950841 & 2874735 \\
\hline ISSSTE & 686426 & 779410 & $83 \mid 447$ & 839099 & 818368 & 900942 & 936978 \\
\hline Pemex & 70304 & 62209 & 58525 & 58237 & 41076 & 66645 & 57338 \\
\hline SDN & 105648 & 13913 & 37960 & 7373 & 14312 & 30026 & 31527 \\
\hline SM & 4128 & 5597 & 5648 & 5912 & 8000 & 7463 & 8209 \\
\hline Usuarias activas de métodos de planificacion familiar & 7494964 & 8019840 & 8362587 & 8465037 & 8833863 & 9168807 & 9523093 \\
\hline Población abierta & 2334482 & 2717377 & 3004577 & 3250285 & 3426129 & 3668786 & 3880735 \\
\hline SSA y SS de entidad federativa ${ }^{\ddagger}$ & I 844567 & 2030633 & 2198566 & 2381428 & 2472872 & 2623024 & 2764300 \\
\hline DDF & 33148 & 30636 & 31092 & - & - & - & - \\
\hline IMSS-Solidaridad & 456767 & 656108 & 774919 & 868857 & 953257 & 1045762 & 1116435 \\
\hline Población derechohabiente & 5160482 & 5302463 & 5358010 & 5214752 & 5407734 & 5500021 & 5642358 \\
\hline IMSS & 4347 I43 & $4465 \quad 177$ & 440457 I & 4242874 & 4379555 & 4408534 & 4496705 \\
\hline ISSSTE & 678694 & $738 \quad 136$ & 798001 & 846286 & 901631 & 953833 & 1005395 \\
\hline Pemex & 70509 & 69220 & 69817 & 70256 & 80728 & 75792 & 74856 \\
\hline SDN & 60319 & 25065 & 79881 & 47440 & 38931 & 52903 & 55548 \\
\hline SM & 3817 & 4865 & 5740 & 7896 & 6889 & 8959 & 9854 \\
\hline Nuevas aceptantes de métodos de planificación familiar & 2433668 & 2494582 & 2566947 & 2485733 & 2523430 & 2523647 & 2572299 \\
\hline Población abierta & 996665 & I 147599 & I 277303 & 1272662 & I 256863 & 1304566 & I 363884 \\
\hline SSA y SS de entidad federativa ${ }^{\ddagger}$ & 717663 & 822883 & 873382 & 916272 & 892763 & 932852 & 991100 \\
\hline DDF & 28950 & 32734 & 31628 & - & - & - & - \\
\hline IMSS-Solidaridad & 250052 & 291982 & 372293 & 356390 & 364100 & 371714 & 372784 \\
\hline Población derechohabiente & I 437003 & I 346983 & I 289644 & $|2| 307 \mid$ & I 266567 & 1219081 & I 208415 \\
\hline IMSS & I 246746 & I I7| 056 & I 106657 & | 04249 | & I 088050 & 1032417 & 1012680 \\
\hline ISSSTE & 133469 & 146979 & 154837 & 149053 & 165070 & 170250 & 177061 \\
\hline Pemex & 9323 & 10533 & 10051 & 10368 & 8423 & 8085 & 9492 \\
\hline SDN & 45329 & 15316 & 15440 & 9003 & 2424 & 4968 & 5485 \\
\hline & & & & & 0 & & \\
\hline
\end{tabular}

* Cifras estimadas

\# Hasta 1996 sólo incluye información de la SSA

ND: no disponible

SSA: Secretaría de Salud; DDF: Departamento del Distrito Federal; IMSS-Solidaridad: Instituto Mexicano del Seguro Social Régimen de Solidaridad; IMSS: Instituto Mexicano del Seguro Social Régimen de Seguridad Social; ISSSTE: Instituto de Seguridad y Servicios Sociales de los Trabajadores del Estado; Pemex: Petróleos Mexicanos; SDN: Secretaría de la Defensa Nacional; SM: Secretaría de Marina

Fuente: Secretaría de Salud, a través de la información proporcionada por las instituciones del Sistema Nacional de Salud 


\section{Indicadores de eficiencia y productividad del Sistema Nacional de Salud. México, 1989-2000 (NÚMERO)}

Concepto

1994

1995

1996

1997

1998

$1999 \quad 2000^{*}$

Recursos por cada 100 mil habitantes ${ }^{\ddagger}$

Humanos

\begin{tabular}{llllllll} 
Médicos & 118.8 & 120.5 & 122.0 & 127.5 & 131.1 & 131.4 & 131.0 \\
\hline Enfermeras & 169.1 & 169.9 & 170.9 & 172.5 & 178.8 & 181.5 & 180.4
\end{tabular}

Físicos

\begin{tabular}{|c|c|c|c|c|c|c|c|}
\hline Consultorios & 45.0 & 45.4 & 45.7 & 47.9 & 48.5 & 48.9 & 49.6 \\
\hline Camas & 74.1 & 73.4 & 72.7 & 74.0 & 75.4 & 75.1 & 75.9 \\
\hline Quirófanos & 2.5 & 2.4 & 2.4 & 2.5 & 2.6 & 2.6 & 2.7 \\
\hline Gabinetes de radiología & 2.4 & 2.4 & 2.5 & 2.5 & 2.6 & 2.6 & 2.6 \\
\hline Laboratorios & 1.6 & 1.6 & 1.6 & 1.7 & 1.7 & 1.8 & 1.8 \\
\hline
\end{tabular}

Servicios proporcionados por cada 1000 habitantes $^{\ddagger}$

\begin{tabular}{|c|c|c|c|c|c|c|c|}
\hline Consultas externas generales & I 255.7 & I 331.6 & I 369.8 & $|45| .3$ & $15 \mid 4.3$ & 1608.6 & I 684.6 \\
\hline Consultas de especialidad & 289.9 & 303.6 & 316.7 & 331.6 & 329.5 & 339.4 & 342.3 \\
\hline Egresos hospitalarios & 41.3 & 41.5 & 41.1 & 42.0 & 41.4 & 42.4 & 43.0 \\
\hline Intervenciones quirúrgicas & 24.2 & 25.2 & 25.7 & 26.1 & 26.7 & 27.2 & 26.8 \\
\hline Estudios de radiología & 159.8 & 156.8 & 158.2 & 162.9 & 164.6 & 167.6 & 183.4 \\
\hline Estudios de laboratorio & I 430.9 & | 454.2 & | 474.1 & | 543.0 & | 577.2 & I 649.5 & I 679.7 \\
\hline
\end{tabular}

Total de consultas externas/total de médicos

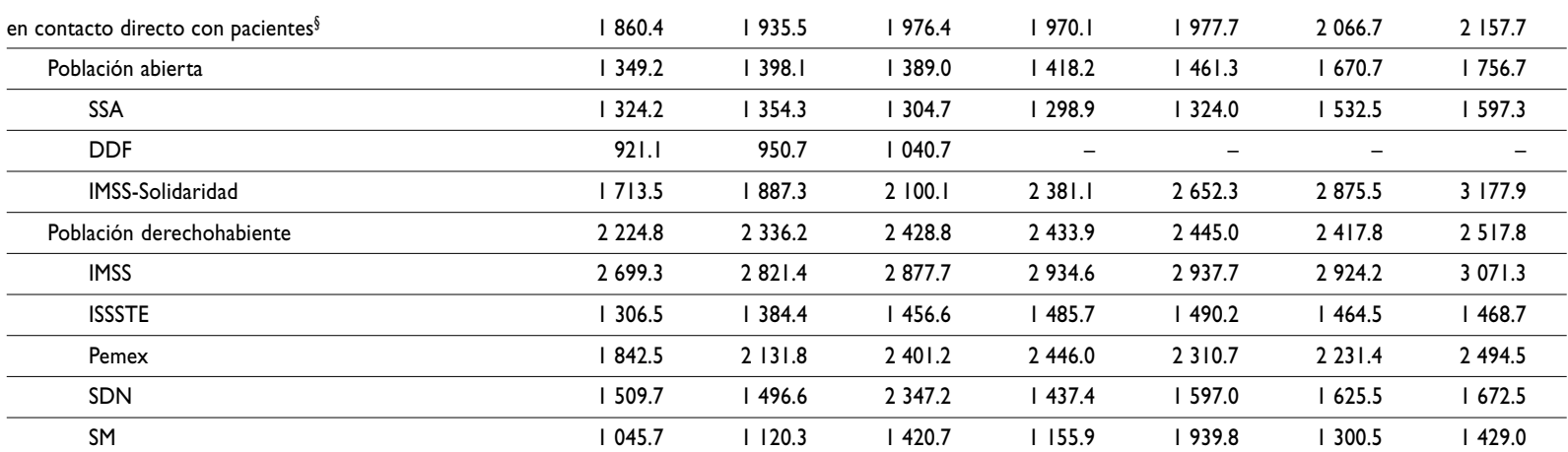

Productividad diaria de los servicios

Consultas externas otorgadas por médico

\begin{tabular}{|c|c|c|c|c|c|c|c|}
\hline General & 14.3 & 14.8 & 15.4 & 15.9 & 15.9 & 16.5 & 17.4 \\
\hline Especialista & 3.0 & 3.2 & 3.4 & 3.4 & 3.2 & 3.2 & 3.3 \\
\hline Consultas generales por consultorio & II.I & 11.6 & 11.9 & 12.0 & 12.4 & 13.1 & 13.5 \\
\hline Intervenciones quirúrgicas por quirófano & 2.7 & 2.8 & 2.9 & 2.9 & 2.8 & 2.8 & 2.8 \\
\hline
\end{tabular}

* Cifras estimadas

¥ Para 1995, 1996 y 1997 cifras revisadas por la dependencia reponsable

$\S$ Incluye el personal médico general, especialista y en formación. A partir de 1997, con motivo de la descentralización se incorporan los datos de los estados del DDF, por lo cual no aparece información de este último, en el renglón en los años de 1997, 1998, 1999 y 2000

* Se consideraron 252 días hábiles, excepto en intervenciones quirúrgicas por quirófano donde se consideraron 365 días hábiles

Fuente: Secretaría de Salud, México 


\section{Cuadro VIII}

\section{Población legal o potencial. Sistema Nacional de Salud, México, 1994-2000}

\begin{tabular}{|c|c|c|c|c|c|c|c|}
\hline Concepto & 1994 & 1995 & 1996 & 1997 & 1998 & 1999 & $2000 *$ \\
\hline Población abierta & 43028182 & 44856068 & 47654693 & 46365853 & 48634302 & 50804634 & 52898398 \\
\hline SSA y SS de entidad federativa ${ }^{\ddagger}$ & 29245000 & 30635000 & 32917250 & 35463500 & 37837500 & 39795000 & $4|88| 500$ \\
\hline DDF & 3464000 & 3681018 & 3698740 & - & - & - & - \\
\hline IMSS-Solidaridad & 10319182 & 10540050 & 11038703 & 10902353 & 10796802 & 11009634 & 11016898 \\
\hline Población derechohabiente & 46884928 & 44614259 & 47650679 & 50165592 & 52937624 & 55779736 & 58611809 \\
\hline IMSS & 36553822 & 34323844 & 37260967 & $3946 \mid 964$ & $4|94| 674$ & $44557 \quad 157$ & $4710044 I$ \\
\hline ISSSTE $^{\S}$ & 9095949 & 9240003 & 9305689 & 9465895 & 9718148 & 9896695 & $10|4| 148$ \\
\hline Pemex & 695565 & 518552 & 539521 & 597078 & 627491 & 603879 & 620069 \\
\hline SDN & 316587 & 315550 & 361344 & 456683 & 456683 & 489477 & 494371 \\
\hline SM & 223005 & 216310 & 183158 & 183972 & 193628 & 232528 & 255780 \\
\hline
\end{tabular}

* Cifras estimadas

\# Hastal996 sólo incluye información de la SSA

¥ Incluye población legal en el extranjero

SSA: Secretaría de Salud; DDF: Departamento del Distrito Federal; IMSS-Solidaridad: Instituto Mexicano del Seguro Social Régimen de Solidaridad; IMSS: Instituto Mexicano del Seguro Social Régimen de Seguridad Social; ISSSTE: Instituto de Seguridad y Servicios Sociales de los Trabajadores del Estado; Pemex: Petróleos Mexicanos; SDN: Secretaría de la Defensa Nacional; SM: Secretaría de Marina

Fuente: Secretaría de Salud, a través de la información proporcionada por las instituciones del Sistema Nacional de Salud 
Cuadro IX

Principales causas de mortalidad por sectores de la población.

Sistema Nacional de Salud, México, 1994-2000

\begin{tabular}{|c|c|c|c|c|c|c|c|}
\hline Concepto & 1994 & 1995 & 1996 & 1997 & 1998 & 1999 & $2000 *$ \\
\hline General $^{\ddagger}$ & 419074 & 430278 & 436321 & 440437 & 444665 & 443950 & 454473 \\
\hline Enfermedades del corazón, excepto fiebre reumática & 59371 & 68058 & 64218 & 66809 & 69513 & 69190 & 71277 \\
\hline Accidentes, envenenamientos y violencias & 58600 & 56919 & 55837 & 56032 & 56022 & 54559 & 54249 \\
\hline Tumores malignos & 46423 & 48292 & 49916 & 51254 & 52670 & 53662 & 55629 \\
\hline Diabetes mellitus & 30324 & 33316 & 34865 & 36027 & 41832 & 45632 & 45827 \\
\hline Enfermedad cerebrovascular & 22666 & 23400 & 24344 & 24689 & 25050 & 25836 & 26271 \\
\hline Cirrosis hepática $^{\mathrm{a}}$ & 20799 & 21245 & 21753 & 22865 & 27211 & 27040 & 29234 \\
\hline Neumonías, influenza y otras infecciones respiratorias agudas & 21267 & 21707 & 22493 & 21596 & 17657 & 16674 & 16946 \\
\hline Ciertas causas de morbilidad y mortalidad perinatal ${ }^{\emptyset}$ & 20584 & 20503 & 19703 & 19821 & 19879 & 19268 & 19131 \\
\hline Enfermedades infecciosas intestinales & 10082 & 9585 & 8359 & 7426 & 6668 & 5622 & 4528 \\
\hline Avitaminosis y otras deficiencias nutricionales & 9585 & 10162 & 10269 & 10157 & 10492 & 9776 & 10675 \\
\hline Las demás causas & 119373 & 117091 & 124564 & 123761 & $11767 \mid$ & 116691 & 120706 \\
\hline Materna & 1409 & I 454 & | $29 \mid$ & 1266 & 1415 & 1400 & | 291 \\
\hline Hemorragias del embarazo y del parto & 340 & 343 & 267 & 249 & 243 & 172 & 148 \\
\hline Sepsis del parto y del puerperio & 57 & 58 & 39 & 35 & 50 & 49 & 41 \\
\hline Otros abortos y los no especificados $\S$ & 95 & 117 & 87 & 107 & 110 & 93 & 93 \\
\hline Toxemia del embarazo y puerperio $^{\Delta}$ & 386 & 411 & 444 & 415 & 474 & 472 & 508 \\
\hline Otras complicaciones del embarazo, parto y puerperio & 531 & 525 & 454 & 460 & 538 & 614 & 501 \\
\hline Infantil ${ }^{\#}$ & 49305 & 48023 & 45707 & 44377 & 42183 & 40283 & 38023 \\
\hline Ciertas afecciones originadas en el periodo perinatal ${ }^{\otimes}$ & 20584 & 20503 & 19703 & 19821 & 19879 & 19268 & 19132 \\
\hline Anomalías congénitas & 7274 & 7383 & 7147 & 7214 & 7612 & 7346 & 7514 \\
\hline Neumonías, influenza y otras infecciones respiratorias agudas & 7689 & 6955 & 6647 & 6218 & 4639 & 4227 & 3477 \\
\hline Enfermedades infecciosas intestinales & 3925 & 3500 & 2915 & 2619 & 2270 & 1996 & 1300 \\
\hline Avitaminosis y otras deficiencias nutricionales & 1454 & 1463 & 1326 & 1202 & I 278 & 1052 & 1054 \\
\hline Bronquitis, enfisema y asma & 662 & 603 & 586 & 484 & 137 & 132 & 91 \\
\hline Las demás causas & 7717 & 7616 & 7383 & 6819 & 6368 & 6262 & 5455 \\
\hline Prescolar ${ }^{\&}$ & 10667 & 10680 & 10375 & 9371 & 8541 & 7774 & 7286 \\
\hline Enfermedades infecciosas intestinales & 1495 & 1364 & 1339 & $|06|$ & 929 & 769 & 596 \\
\hline Neumonías, influeza y otras infecciones respiratorias agudas & 1669 & 1694 & 1498 & I 259 & 1068 & 932 & 697 \\
\hline Accidentes, envenenamientos y violencias & 2417 & 2242 & 2136 & 2125 & 1992 & 2070 & 1777 \\
\hline Tosferina & 9 & 3 & 2 & 3 & 0 & 0 & 0 \\
\hline Avitaminosis y otras deficiencias nutricionales & 606 & 713 & 798 & 653 & 609 & 467 & 607 \\
\hline Sarampión & I & 0 & 0 & 0 & 0 & 0 & 0 \\
\hline Las demás causas & 4470 & 4664 & 4602 & 4270 & 3943 & 3536 & 3609 \\
\hline Escolar $\neq$ & 8015 & 8039 & 8091 & 7956 & 7882 & 7483 & 7758 \\
\hline Enfermedades infecciosas intestinales ${ }^{\infty}$ & 301 & 303 & 264 & 247 & 199 & 172 & 143 \\
\hline Neumonías, influenza y otras infecciones respiratorias agudas & 397 & 427 & 407 & 339 & 267 & 262 & 202 \\
\hline Accidentes, envenenamientos y violencias & 3321 & 3133 & 3114 & 3207 & 3271 & 3067 & 3267 \\
\hline Enfermedades del corazón, excepto fiebre reumática & 148 & 256 & 145 & 150 & 149 & 142 & 93 \\
\hline Tumores malignos & 899 & 930 & I $05 \mid$ & 1017 & 1089 & 1049 & 1173 \\
\hline Sarampión & 1 & 0 & 0 & 0 & 0 & 0 & 0 \\
\hline Las demás causas & 2948 & 2990 & 3110 & 2996 & 2907 & 2791 & 2880 \\
\hline
\end{tabular}

(Continúa) 
Cuadro IX

(Continuación)

\begin{tabular}{|c|c|c|c|c|c|c|c|}
\hline Productiva $^{\circ}$ & $16147 \mid$ & 163184 & 162252 & 165779 & 168519 & 167105 & 170355 \\
\hline Enfermedades infecciosas intestinales & 1700 & 1720 & 1404 & 1212 & 1052 & 888 & 606 \\
\hline Neumonías, influenza y otras infecciones respiratorias agudas & 3160 & 3408 & 3512 & 3474 & 2753 & 2679 & 2754 \\
\hline Accidentes, envenenamientos y violencias & 43266 & 42093 & 40962 & 41156 & 40997 & 39449 & 39461 \\
\hline Enfermedades del corazón, excepto fiebre reumática & 15913 & 17203 & 16162 & $|735|$ & 16941 & 17594 & 17248 \\
\hline Tumores malignos & 20994 & 21610 & 22197 & 22917 & 23664 & 23832 & 24904 \\
\hline Cirrosis hepática & 15109 & 14930 & 15008 & 15769 & 18413 & 18345 & 19336 \\
\hline Tuberculosis, todas formas & 2822 & 2771 & 2643 & 2469 & 2369 & 2111 & 2056 \\
\hline Enfermedades cerebrovasculares & 5886 & 5880 & 6005 & 6181 & 6183 & 6352 & 6379 \\
\hline Complicaciones del embarazo, parto y puerperio & 400 & I 444 & 1285 & 1256 & 402 & 1399 & 1286 \\
\hline Diabetes mellitus & 12717 & 14005 & 14283 & 14967 & 17337 & 18647 & 18832 \\
\hline Las demás causas & 38504 & 38120 & 38791 & 39027 & 37408 & 35809 & 37493 \\
\hline $\begin{array}{l}\text { * Cifras estimadas } \\
\text { \# El total no coincide con las sumas de las causas de mortalidad, } \\
\S \text { Comprende todos los abortos } \\
\text { \# Corresponde a la población menor de un año } \\
\text { \& Corresponde a la población de I a } 4 \text { años } \\
\text { \# Corresponde a la población de } 5 \text { a I } 4 \text { años } \\
\infty \text { Incluye defunciones por fiebre tifoidea, paratifoidea y otras salm } \\
\text { - Corresponde a la población de I5 a } 64 \text { años } \\
\text { Incluye tétanos neonatal A33.X de la Lista Detallada de la CIE-I } \\
\text { "A partir de } 1998 \text { se tomaron las enfermedades del hígado } \\
\triangle \text { A partir de } 1998 \text { se consideraron: edema, proteinuria y trastor }\end{array}$ & osis & las defun & ones del g & po de eda & posprodu & & \\
\hline
\end{tabular}

